\title{
Erratum: Floquet Weyl semimetals in light-irradiated type-II and hybrid line-node semimetals [Phys. Rev. B 97, 155152 (2018)]
}

\author{
Rui Chen, Bin Zhou, and Dong-Hui Xu
}

(Received 7 May 2019; published 12 July 2019)

DOI: 10.1103/PhysRevB.100.049901

There are some errors in this paper. Equations (3), (4), and (15) are incorrect. Some terms were overlooked by mistake, and the tilt ratio Eq. (4) of line-node semimetals (LNSMs) is not defined precisely. The conclusions of the paper remain valid, but the following corrections should be made:

The energy dispersion around $\mathbf{k}_{0}$ in Eq. (3) should be

$$
\begin{aligned}
E_{ \pm}^{0}\left(\mathbf{q}=\mathbf{k}-\mathbf{k}_{0}\right) & =\boldsymbol{w} \cdot \mathbf{q} \pm \sqrt{\left(\xi_{x} q_{x}+\xi_{z} q_{z}\right)^{2}+\xi_{y}^{2} q_{y}^{2}} \\
& =T(\mathbf{q}) \pm U(\mathbf{q}),
\end{aligned}
$$

where $\xi_{x}^{2}=4 m_{0} m_{x} C_{\theta}^{2}, \xi_{y}^{2}=v_{y}^{2}, \xi_{z}^{2}=4 m_{0} m_{z} S_{\theta}^{2}$, and $\boldsymbol{w}=2\left(c_{x} C_{\theta} \sqrt{m_{0} / m_{x}}, 0, c_{z} S_{\theta} \sqrt{m_{0} / m_{z}}\right)$. In the original paper, the term $2 \xi_{x} q_{x} \xi_{z} q_{z}$ under the square root was missing. In addition, the coefficients $\xi_{x, y, z}$ and $\boldsymbol{w}$ are corrected by multiplying factors 4 and 2 , respectively.

Around point $\mathbf{k}_{0}$ on the nodal ring, we obtain a two-dimensional Dirac cone when the small vector $\mathbf{q}$ is confined on the transverse plane formed by the in-plane normal line to the nodal ring and the line along the $k_{y}$ axis. From Eq. (3), we can see that the tilt is most effective when $\mathbf{q}$ is along the direction of normal-vector $\mathbf{k}_{n}=\left(m_{x} k_{0 x}, m_{z} k_{0 z}\right)$. Then, the tilt ratio Eq. (4) should be [1]

$$
F_{\theta}=\left|\frac{T\left(\hat{\mathbf{k}}_{n}\right)}{U\left(\hat{\mathbf{k}}_{n}\right)}\right|=\left|\frac{c_{x} C_{\theta}^{2}+c_{z} S_{\theta}^{2}}{m_{x} C_{\theta}^{2}+m_{z} S_{\theta}^{2}}\right|,
$$

where $\hat{\mathbf{k}}_{n}=\mathbf{k}_{n} /\left|\mathbf{k}_{n}\right|$ is the normal unit vector on the $k_{x}-k_{z}$ plane. In the original paper, we took the tilt direction along $\boldsymbol{w}$ by mistake. However, around $\mathbf{k}_{0}$, to better define the tilt ratio, $\mathbf{q}$ should be confined on the plane formed by $\mathbf{k}_{n}$ and $\mathbf{k}_{y}$ since we can think of the nodal ring as a collection of nodal points. The condition for a type-II nodal ring now requires $\left|c_{x} / m_{x}\right|>1,\left|c_{z} / m_{z}\right|>1$, and $c_{x} c_{z}>0$. In the original paper, we overlooked the constraint $c_{x} c_{z}>0$. The condition for a type-I nodal ring is the same as that in the original paper. In the rest of the cases, the Hamiltonian gives a hybrid nodal ring.

In Eq. (15), the tilt ratio of the induced Weyl semimetal phase for light propagating on the $x-z$ plane should be

$$
F_{\psi}=\frac{\lambda_{x}^{2}+\lambda_{z}^{2}}{\sqrt{L^{2}\left(C_{\psi} m_{x} \lambda_{x}-S_{\psi} m_{z} \lambda_{z}\right)^{2}+4 m_{x} m_{z} \tilde{m}_{0}^{\prime \prime}\left(S_{\psi} \lambda_{x}+C_{\psi} \lambda_{z}\right)^{2} / K}} .
$$

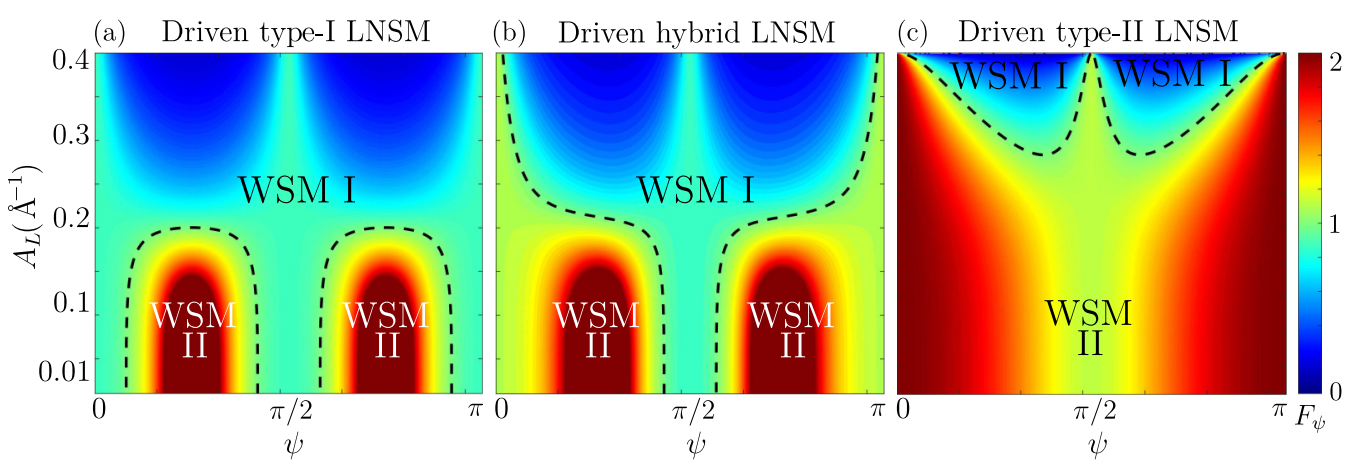

FIG. 3. Phase diagrams of driven LNSMs in the $\left(A_{L}, \psi\right)$ phase space. The color bar indicates the tilt ratio $F_{\psi}$. The phase boundaries are marked by the dashed lines, corresponding to $F_{\psi}=1$. The model parameters are taken to be (a) $-c_{x}=c_{y}=c_{z}=3.5 \mathrm{eV} \AA^{2},(\mathrm{~b})-c_{x}=$ $c_{y}=3.5$ and $c_{z}=4.5 \mathrm{eV} \AA^{2}$, (c) $c_{x}=c_{y}=4.5$ and $c_{z}=8 \mathrm{eV} \AA^{2}$. The common parameters are $m_{x}=m_{y}=m_{z}=4 \mathrm{eV} \AA^{2}, m_{0}=1 \mathrm{eV}, v_{y}=1 \mathrm{eV} \AA$, and $\hbar \omega=0.1 \mathrm{eV}$. 
The term $8 m_{x} m_{z} \tilde{m}_{0}^{\prime \prime} S_{\psi} \lambda_{x} C_{\psi} \lambda_{z} / K-2 L^{2} C_{\psi} m_{x} \lambda_{x} S_{\psi} m_{z} \lambda_{z}$ of the denominator was missing in the original paper. Correspondingly, Fig. 3 should be updated to the current version. Note that the phase boundaries in the revised version of Figs. 3(a) and 3(b) remain almost unchanged, but the value distribution of the tilt ratio $F_{\theta}$ is different from the previous one. In the revised version of Fig. 3(c), the phase boundary varies a lot.

[1] S. Li, Z.-M. Yu, Y. Liu, S. Guan, S.-S. Wang, X. Zhang, Y. Yao, and S. A. Yang, Phys. Rev. B 96, 081106(R) (2017). 